\title{
Adjuvant Biological Therapies in Chronic Leg Ulcers
}

\author{
Natalia Burgos-Alonso ${ }^{1,2}$, Igone Lobato ${ }^{3}$, Igone Hernández ${ }^{3}$, Kepa San Sebastian ${ }^{1}$, \\ Begoña Rodríguez ${ }^{4}$, Gontzal Grandes ${ }^{1}$ and Isabel Andia ${ }^{5, *}$ \\ 1 Primary Care Research Unit of Bizkaia, BioCruces Health Research Institute, 48014 Bilbao, Spain; \\ natalia.burgos@ehu.es (N.B.-A.); ksansebastian@gmail.com (K.S.S.); \\ gonzalo.grandesodriozola@osakidetza.eus (G.G.) \\ 2 Preventive Medicine and Public Health Department, Faculty of Medicine and Odontology, Universidad del \\ País Vasco/Euskal Herriko Unibertsitatea UPV/EHU, University of the Basque Country, 48940 Lejona, Spain \\ 3 Enkarterrri-Ezkerraldea-Cruces Health Region, Basque Health Service (Osakidetza), 48903 Barakaldo, Spain; \\ igone.lobatogarcia@osakidetza.eus (I.L.); igone.hernandezcabezas@osakidetza.eus (I.H.) \\ 4 Bilbao-Basurto Health Region, Basque Health Service (Osakidetza), 48014 Bilbao, Spain; \\ mariabegona.rodriguezrodriguez@osakidetza.eus \\ 5 Regenerative Medicine Laboratory, BioCruces Health Research Institute, Cruces University Hospital, \\ 48903 Barakaldo, Spain \\ * Correspondence: Isabel.andiaortiz@osakidetza.eus; Tel.: +34-946-007-964 or +34-609-419-897
}

Received: 3 November 2017; Accepted: 28 November 2017; Published: 28 November 2017

\begin{abstract}
Current biological treatments for non-healing wounds aim to address the common deviations in healing mechanisms, mainly inflammation, inadequate angiogenesis and reduced synthesis of extracellular matrix. In this context, regenerative medicine strategies, i.e., platelet rich plasmas and mesenchymal stromal cell products, may form part of adjuvant interventions in an integral patient management. We synthesized the clinical experience on ulcer management using these two categories of biological adjuvants. The results of ten controlled trials that are included in this systematic review favor the use of mesenchymal stromal cell based-adjuvants for impaired wound healing, but the number and quality of studies is moderate-low and are complicated by the diversity of biological products. Regarding platelet-derived products, 18 controlled studies investigated their efficacy in chronic wounds in the lower limb, but the heterogeneity of products and protocols hinders clinically meaningful quantitative synthesis. Most patients were diabetic, emphasizing an unmet medical need in this condition. Overall, there is not sufficient evidence to inform routine care, and further clinical research is necessary to realize the full potential of adjuvant regenerative medicine strategies in the management of chronic leg ulcers.
\end{abstract}

Keywords: biological therapies; chronic leg ulcer; platelet rich plasma; bone marrow concentrates; stromal vascular fraction; mesenchymal stromal cells

\section{Introduction}

The overwhelming costs of wound care services is rising worldwide [1,2], with the market of wound care products surpassing $\$ 15$ billion according to Global Industry Analysts [3]. Healing chronic wounds is becoming a major health challenge. In particular, chronic wounds in the lower limb represent the largest fraction, with venous and diabetic foot ulcers (DFU)s accounting for $70-90 \%$ of these ulcers [4]. The socioeconomic and biomedical burdens that they represent are worsened by global demographic events, such as the aging population and the pandemic of obesity [5]. The latter is associated with an increased incidence of diabetes and the threat it involves in foot ulcer development. In fact, up to $25 \%$ of diabetic patients will develop a foot ulcer with costs ranging from $\$ 7439$ to $\$ 20,622$ per episode [6]. Furthermore, ulcer chronicity increases the severity and the costs of these conditions. 
Wound care encompasses all elements of wound management, which include the control of underlying conditions, including neuropathy, ischemia, venous hypertension, pressure, and infection. Impaired wound healing can occur even after controlling modifiable risk factors, and adjuvant biological interventions can form part of complex wound management. Actually, prompt wound healing is imperative to prevent irreversible damage. Moreover, the longer it takes to heal an ulcer, the greater the severity and the financial burden [7].

Concepts in wound healing pathophysiology help to determine the choice of therapy and care planning. The involvement of growth factors was acknowledged decades ago, and as a result, recombinant growth factors (rh-GFs) therapies, such as recombinant human epidermal growth factor (rh-EGF) [8], recombinant human fibroblast growth factor (rh-FGF) [9], and recombinant human platelet-derived growth factor (rh-PDGF) [10] have been explored, resulting in specific growth factors in the therapeutic armamentarium, such as Regranex ${ }^{\circledR}$, (becaplermin, rh-PDGF-BB). But, one by one these growth factors cannot fulfill the multiple needs of non-healing tissues. Grounded on a more biomimetic hypothesis, interventions, such as platelet rich plasma (PRP) therapies, are being tested as they deliver a large pool of molecules that are involved in various healing stages, which can be stalled due to different comorbidities. Healing stages susceptible of PRP modulation include hemostasis, inflammation, cell migration and proliferation, extracellular matrix production, and tissue remodeling [11].

Furthermore, the use of cellular products, in order to address a potential deficiency of competent cells, is under scrutiny. Currently, medical devices and protocols are commercially available to prepare different PRP formulations, and cellular products containing a low number of MSCs, i.e., bone marrow concentrates (BMC), and the stromal vascular fraction (SVF) from adipose tissue [12].

In the context of difficult to heal wounds, regenerative medicine strategies, i.e., PRPs and cell products, may form part of adjuvant interventions in an integral patient management; moreover, taking advantage of using patients' own resources and in order to avoid drug interactions in these otherwise polimedicated patients, it may be possible to prepare PRP, BMC, or SVF for local application in the wound.

Our review aims to synthesize the clinical experience on ulcer management using two categories of biological adjuvants. Firstly, we have explored the use of point of care MSCs' related products, and second the local application of platelet derived products. The question that we addressed is as follows: is there any mesenchymal stromal cell or platelet-based regenerative therapy that applied locally, either injected in the wound edges and/or applied topically in the wound bed, can help to heal chronic leg ulcers? The results of controlled trials included in this systematic review favor the use of mesenchymal cell based-adjuvants for impaired wound healing, but the number and quality of studies is moderate-low and complicated by the diversity of biological products. Overall, there is not sufficient evidence to inform routine care. There are 18 controlled studies that are investigating the efficacy of platelet-derived products in lower limb chronic wounds, but the heterogeneity of products and protocols hinders clinically meaningful quantitative synthesis.

\section{Results}

The search resulted on 813 articles, after removing duplicates. After exclusion of 782 articles for the reasons shown in Figure 1, thirty-one articles remained.

Four articles were excluded after reviewing the full text because they involved the treatment of chronic osteomyelitis, pressure ulcers in the trunk, with various anatomical locations or burn injuries. The main characteristics of cell-based and platelet-based studies are summarized Tables 1 and 2 [13-40], respectively. 
Table 1. Summary of included studies.

\begin{tabular}{|c|c|c|c|c|c|c|}
\hline Reference (Year) & $\begin{array}{l}\text { Study Design } \\
\text { Experimental/ } \\
\text { Control Group } \\
\end{array}$ & Patient Population & Selection Criteria & $\begin{array}{c}\text { Biological Intervention and Control } \\
\text { Management }\end{array}$ & Outcomes/Follow-up & Differences and Statistical Results \\
\hline Dash (2009) [13] & $\begin{array}{c}\text { RCT } \\
\operatorname{Exp} N=12 \\
\text { CTR } N=12\end{array}$ & $\begin{array}{l}\text { Diabetic foot and } \\
\text { Burger disease }\end{array}$ & Chronicity $>1$ month & $\begin{array}{l}\text { Exp: BM-MSCs CD } 90^{+}, \mathrm{CD} 105^{+}, \mathrm{CD} 34^{-}, \\
\text {expanded for } 5 \text { passages or more } \\
\text { CTR: standard wound dressing }\end{array}$ & $\begin{array}{l}\text { Pain-free walking distance and } \\
\text { reduction in ulcer size/12 weeks }\end{array}$ & $\begin{array}{l}\text { Cell implant group better than } \\
\text { control in pain-free walking and } \\
\text { reduction in ulcer area }\end{array}$ \\
\hline Dubsky (2013) [14] & $\begin{array}{c}\text { Consecutive patients } \\
\text { (non-randomized) } \\
\text { Three armed } \\
\text { Exp1, } N=17 \text { patients; } \\
\text { Exp2, } N=11 \text { patients } \\
\text { CTR } N=22 \\
\end{array}$ & Diabetic foot disease & $\begin{array}{l}\text { Critical limb ischemia PEDIS } 3 \\
\mathrm{TcPO}_{2} 30 \mathrm{mmHG} \text { or ABI }<0.6\end{array}$ & $\begin{array}{c}\text { Exp1: BMC } \\
\text { Exp2: PBMNC } \\
\text { (after G-CSF mobilization) } \\
\text { CTR: standard care }\end{array}$ & $\begin{array}{l}\text { Rate of major amputation and } \\
\mathrm{TcO}_{2} / 6 \text { months } \\
\text { Lost to follow-up: } 3\end{array}$ & $\begin{array}{c}\text { Amputations: } 11 \% \text { in the SCT vs. } \\
50 \% \text { CTR }(p=0.0032) \\
\text { No differences } \\
\text { between BMC and PBMNC }\end{array}$ \\
\hline Han (2009) [15] & $\begin{array}{c}\text { RCT } \\
\text { Exp } N=26 \text { patients } \\
\text { CTR } N=26\end{array}$ & Diabetic ulcers & Chronicity $>6$ weeks & $\begin{array}{l}\text { Exp1: SVF+ (fibrinogen/fibrin) } \\
\text { C: (fibrinogen/fibrin) }\end{array}$ & $\begin{array}{l}\text { Ulcer size/ } 8 \text { weeks } \\
\text { Lost to follow-up: } 2\end{array}$ & $\begin{array}{l}100 \% \text { complete wound healing in } \\
\text { intervention and } 62 \% \text { complete } \\
\text { healing in control group }\end{array}$ \\
\hline Jain (2011) [16] & $\begin{array}{c}\text { RCT } \\
\text { Exp, } N=25 \text { patients } \\
\text { CTR } N=23\end{array}$ & $\begin{array}{l}\text { Chronic lower limb } \\
\text { foot in patients with } \\
\text { diabetes mellitus }\end{array}$ & Chronicity $>3$ months & $\begin{array}{l}\text { Exp: BMC injection } \\
\text { CTR: peripheral blood injection }\end{array}$ & $\begin{array}{c}\text { Complete closure } \\
\text { Area reduction } \\
\text { Wound suitable for surgery }\end{array}$ & $\begin{array}{c}40 \% \text { ulcer healed in Exp vs. } 29 \% \text { in } \\
\text { CTR } p<0.05 \\
\text { Area reduction: } \\
\text { Exp: } 36 \% / \text { SD0.48 } \\
\text { CTR: } 27.32 \% \text { SD0.32 } \\
\text { No differences between groups at } \\
3 \text { months } \\
\text { Exp } N=3 \text { vs. CTR } N=1 \text { had skin } \\
\text { grafts } / 3 \text { months } \\
\text { Lost to follow-up: } 2\end{array}$ \\
\hline Kirana (2012) [17] & $\begin{array}{c}\text { RCT } \\
\text { Exp } 1, N=12 \text { patients } \\
\operatorname{Exp} 2 N=12 \\
\text { CTR } N=6\end{array}$ & Diabetic ulcers & Chronicity $>6$ weeks & $\begin{array}{c}\text { Exp1: BMC } \\
\text { Exp2: TRC/BM-MSC expanded } \\
\text { enriched in CD } 90^{+} \text {cells } \\
\text { CTR: high } \mathrm{n}^{\circ} \text { of drop outs, } \\
4 / 6 \text { led to exclusion }\end{array}$ & $\begin{array}{c}\text { Complete healing/8 week. Lost to } \\
\text { follow-up: } 2 \text { Secondary endpoints: } \\
\text { time to complete healing, } \mathrm{n}^{\circ} \text { major } \\
\text { amputation improvement in ABI, } \\
\text { TcPO2, BOLD }\end{array}$ & $\begin{array}{l}22 \text { patients received cell treatment. } \\
\text { One patient in the TRC group and } \\
\text { two in the BMC group did not show } \\
\text { wound healing during follow up, } \\
18 \text { patients healed }\end{array}$ \\
\hline $\mathrm{Lu}(2011)[18]$ & $\begin{array}{l}\text { RCT, three } \\
\text { armed-study } \\
\text { Exp1, } N=20 \text { patients; } \\
\text { Exp2, } N=21 \text { patients } \\
\text { CTR: contra-lateral } \\
\text { ulcer, } N=41\end{array}$ & $\begin{array}{l}\text { Diabetic patients with } \\
\text { CLI and foot ulcer }\end{array}$ & $\begin{array}{l}\text { Bilateral critical limb ischemia } \\
\text { (ABI 0.30-0.60) }\end{array}$ & $\begin{array}{l}\text { Exp1: expanded BM-MSC with } \\
\text { autologous serum } \\
\text { Exp2: BMC } \\
\text { CTR: normal saline }\end{array}$ & $\begin{array}{l}\text { Ulcer healing rate, pain at rest and } \\
\text { at walking, } \mathrm{ABI}, \mathrm{TcO} \mathrm{T}_{2} \text {, } \\
\mathrm{MRA} / 24 \text { weeks } \\
\text { Lost to follow-up: } 4\end{array}$ & $\begin{array}{l}\text { BM-MSC better than BMC in pain a } \\
\text { walking }(p=0.040), \mathrm{ABI} p=0.017 \\
\mathrm{TcO}_{2} p=0.001, \mathrm{MRA} p=0.018 \\
\text { Cell treated ulcers better than } \\
\text { controls in all outcome measures. } \\
\text { After } 6 \text { weeks the number of healing } \\
\text { ulcers in Exp1 was significantly } \\
\text { higher than Exp2 }\end{array}$ \\
\hline Marino (2013) [19] & $\begin{array}{l}\text { Cohort study } \\
\operatorname{Exp} N=10 \\
\text { CTRL } N=10\end{array}$ & $\begin{array}{c}\text { Arteriopathic } \\
\text { patients, } 18 / 20 \text { had } \\
\text { Diabetes mellitus } \\
\text { type 2, five had heart } \\
\text { disease and } 6 \text { had } \\
\text { chronic obstructive } \\
\text { pulmonary disease }\end{array}$ & $\begin{array}{c}\mathrm{ABI}=0.3-0.4 \text {. all patients } \\
\text { underwent revascularization } \\
\text { procedure without healing and } \\
\text { hyperbaric chamber and oxygen } \\
\text { therapy for } 6 \text { months }\end{array}$ & $\begin{array}{l}\text { Exp1: SVF, Celution system }{ }^{\circledR}(5 \mathrm{~mL}) \\
\text { cells injected, in all directions, at the } \\
\text { edge of the ulcer, depth } 1 \mathrm{~cm} \\
\text { CTR: SVF untreated }\end{array}$ & $\begin{array}{l}\text { Complete closure (primary) } \\
\text { Decrease in diameter and } \\
\text { depth (secondary) }\end{array}$ & $\begin{array}{c}\text { Follow-up: } 4,10,20,60 \text { and } 90 \text { days } \\
\text { Complete healing in six of } \\
10 \text { patients } \\
\text { Four patients did not respond to } \\
\text { SVF treatment }\end{array}$ \\
\hline
\end{tabular}


Table 1. Cont.

\begin{tabular}{|c|c|c|c|c|c|c|}
\hline Reference (Year) & $\begin{array}{l}\text { Study Design } \\
\text { Experimental/ } \\
\text { Control Group } \\
\end{array}$ & Patient Population & Selection Criteria & $\begin{array}{c}\text { Biological Intervention and Control } \\
\text { Management }\end{array}$ & Outcomes/Follow-up & Differences and Statistical Results \\
\hline Procházka (2010) [20] & $\begin{array}{c}\text { RCT } \\
\operatorname{Exp} N=42 \\
\text { CTR } N=54\end{array}$ & $\begin{array}{l}96 \text { patients with } \\
\text { diabetes except } 5 \text { in } \\
\text { the experimental } \\
\text { group; all with CLI } \\
\text { and foot ulcer }\end{array}$ & $\begin{array}{l}\text { chronic and critical limb } \\
\text { ischemia according to the TASC } \\
\text { classificiction Rutherford 4-6, } \\
\text { Fontaine IV }\end{array}$ & $\begin{array}{l}\text { Exp: BMC injection } \\
\text { CTR: conventional treatment } 40 \\
\text { injections each } 1 \mathrm{~mL} \text { into } \\
\text { the ischemic limb }\end{array}$ & $\begin{array}{l}\text { Major limb amputation during } \\
120 \text { days } / 13 \text { patients died of } \\
\text { causes unrelated to therapy }\end{array}$ & $\begin{array}{l}\text { Amputation rate } \\
\text { Exp: } 21 \% \\
\text { CTR: } 44 \%\end{array}$ \\
\hline Raposio (2016) [21] & $\begin{array}{c}\text { RCT } \\
\text { Exp, } N=16 \text { patients } \\
(21 \text { ulcers }) \\
\text { CTR, } N=24 \text { patients } \\
(31 \text { ulcers })\end{array}$ & $\begin{array}{l}\text { Chronic skin ulcers } \\
\text { (diabetic, post-trauma, } \\
\text { arterial, venous) }\end{array}$ & $\begin{array}{l}\text { Ulcer chronicity in the } \\
\text { interventional group: } 10.19(\mathrm{SD}: \\
\text { 4.37) months and } 14.53(9.75) \\
\text { months in the control group }\end{array}$ & $\begin{array}{c}\text { Exp: ePRP:SVF (mechanical disruption) } \\
\text { + PRP (plt: } 4-7 x) \\
\text { CTR: Standard wound care }\end{array}$ & $\begin{array}{l}\text { Wound closure rate } / 18 \text { month } \\
\text { Lost to follow-up: } 0\end{array}$ & $\begin{array}{c}\text { Exp: } 0.2287 \mathrm{~cm} / \text { day vs. CTR: } 0.0890 \\
\mathrm{~cm} / \text { day }(p=0.0257) \text { No matched } \\
\text { groups, baseline differences in ulcer } \\
\text { area (EXP vs. CTR } 29.59 \mathrm{~cm}^{2} \\
\left.\text { vs. } 8.5 \mathrm{~cm}^{2}\right)\end{array}$ \\
\hline Walter (2011) [22] & $\begin{array}{c}\text { RCT } \\
\operatorname{Exp} N=19 \\
\text { CTR } N=21\end{array}$ & $\begin{array}{l}\text { Aterosclerotic } \\
\text { patients }\end{array}$ & $\begin{array}{l}\text { Nonhealing ulcers } \\
\text { (Rutherford class } 5 \text { or } 6 \text { ) }\end{array}$ & $\begin{array}{l}\text { Exp: autologous bone marrow-derived } \\
\text { mononuclear cells (BM-MNC) } \\
\text { CTR: Placebo }\end{array}$ & $\begin{array}{c}\text { Complete } \\
\text { healing/amputation-free } \\
\text { survival/freedom from rest pain } \\
\text { Lost to follow-up: } 12\end{array}$ & $\begin{array}{l}\text { Ulcer area decreased significantly in } \\
\text { the BM-MNC }(p<0.014) \text { but not in } \\
\text { CTR group. Patients in CTR group } \\
\text { switched to BM-MNC treatment and } \\
\text { ulcer area decreased at } 3 \text { months. } \\
\text { Repeated BM-MNC administration } \\
\text { significantly correlated with } \\
\text { complete ulcer healing } \\
\end{array}$ \\
\hline \multicolumn{7}{|c|}{$\begin{array}{l}\mathrm{ABI}=\text { ankle brachial index; } \mathrm{BM}-\mathrm{MNC}=\text { bone marrow derived mononuclear cells (isolated through gradient centrifugation); } \mathrm{BM}-\mathrm{MSC}=\text { bone marrow derived mesenchymal stromal } \\
\text { cells (purified and expanded ex vivo); } \mathrm{BMC}=\text { bone marrow concentrate; } \mathrm{BOLD}=\text { blood oxygen level dependent; } \mathrm{CLI}=\text { critical limb ischemia; } \mathrm{CTR}=\text { control group; } \mathrm{Exp}=\mathrm{Experimental} \\
\text { treatment; } \mathrm{G}-\mathrm{CSF}=\text { granulocyte colony stimulating factor; } \mathrm{PBMNC}=\text { peripheral blood mononuclear cells; } \mathrm{PRP}=\text { platelet rich plasma; } \mathrm{RCT}=\text { randomised controlled trial; } \mathrm{SVF}=\mathrm{stromal} \\
\text { vascular fraction; } \mathrm{TcO}_{2}=\text { transcutaneous oxygen pressure, } \mathrm{TRC}=\text { tissue regenerative cells. }\end{array}$} \\
\hline $\begin{array}{l}\text { Author (Year) } \\
\text { [Reference] }\end{array}$ & $\begin{array}{l}\text { Study Design } \\
\text { Experimental/ } \\
\text { Control Group }\end{array}$ & Patient Population & Chronicity of the Ulcer & $\begin{array}{l}\text { Biological Intervention and Control } \\
\text { Management }\end{array}$ & Outcomes/Follow-Up & Differences and Statistical Results \\
\hline Ahmed (2017) [23] & $\begin{array}{l}\text { RCT } \\
\text { Exp } N=28 \text { patients } \\
\text { CTR } N=28 \text { patients } \\
\text { Matched wounds } \\
\text { between groups }\end{array}$ & DFU 56 patients & $>6$ weeks & $\begin{array}{c}\text { Exp: Autologous gelified PRP (4-5x) } \\
\text { twice weekly } \\
\text { CTR: antiseptic oilment }\end{array}$ & $\begin{array}{l}\text { Ulcer healing, healing rate/ } \\
8 \text { weeks }\end{array}$ & $\begin{array}{l}\text { Exp: } 86 \% \\
\text { CR: } 68 \% \\
\text { Healing rate: Exp: } 0.7 \mathrm{~cm}^{2} / \text { week } \\
\text { CTR: } 0.5 \mathrm{~cm}^{2} / \text { week }\end{array}$ \\
\hline Anitua (2008) [24] & $\begin{array}{c}\text { RCT (pilot) } \\
\text { Exp } N=8 \text { patients } \\
\text { CTR } N=7 \text { patients }\end{array}$ & $\begin{array}{l}64 \% \text { venous, } 29 \% \\
\text { pressure, } 7 \% \text { other } \\
\text { Baseline characteristics } \\
\text { were not similar } \\
\text { between groups }\end{array}$ & $>4$ weeks & $\begin{array}{l}\text { Exp: Autologous gelified PRP }(1.5-2.5 \mathrm{x}) \\
\text { CTR: Conventional treatment }\end{array}$ & $\begin{array}{l}\text { Mean percentage of surface } \\
\text { healed/8 weeks } \\
\text { Lost to follow-up: } 6\end{array}$ & $\begin{array}{c}\text { Exp: } 5 \text { patients } \\
\text { CTR: } 4 \\
\text { Exp: } 72.94 \% \text { (SD: } 22.25 \%) \\
\text { CTR: } 21.48 \% \text { (SD: } 33.56 \%) p<0.05\end{array}$ \\
\hline
\end{tabular}


Table 2. Cont.

\begin{tabular}{|c|c|c|c|c|c|c|}
\hline $\begin{array}{l}\text { Author (Year) } \\
\text { [Reference] }\end{array}$ & $\begin{array}{l}\text { Study Design } \\
\text { Experimental/ } \\
\text { Control Group } \\
\end{array}$ & Patient Population & Chronicity of the Ulcer & $\begin{array}{l}\text { Biological Intervention and Control } \\
\text { Management }\end{array}$ & Outcomes/Follow-Up & Differences and Statistical Results \\
\hline Danielsen (2008) [25] & $\begin{array}{c}\text { RCT } \\
\text { Exp } N=10 \text { patients } \\
\text { CTR } N=10 \text { patients }\end{array}$ & $\begin{array}{l}\text { Graft surgery in } \\
\text { patients with chronic } \\
\text { leg ulcers (evaluation } \\
\text { of meshed autografts } \\
\text { and acute split } \\
\text { thickness donor wounds) }\end{array}$ & Non-reported & $\begin{array}{l}\text { Exp: platelet rich fibrin (Vivostat) } \\
\text { CTR: saline } \\
\text { Platelet rich fibrin sprayed into the } \\
\text { donor and recipient wound plus three } \\
\text { dressings (two different dressings and } \\
\text { one polyurethane closure) }\end{array}$ & $\begin{array}{l}\text { Wound Epithelialization } \\
\text { Immunohistomorphometry } \\
\text { pain } / 20 \text { weeks }\end{array}$ & $\begin{array}{c}\text { Epithelial coverage of donor wounds } \\
\text { did not differ significantly between } \\
\text { platelet-rich fibrin and control on } \\
\text { day } 5 \text { or day } 8\end{array}$ \\
\hline Driver (2006) [26] & $\begin{array}{c}\text { RCT } \\
\text { Exp } N=19 \text { patients } \\
\text { CTR } N=21 \text { patients }\end{array}$ & $\begin{array}{l}72 \text { patients with type } \\
\text { I or II diabetes } \\
\text { Efficacy analysis dropouts }\end{array}$ & $>4$ weeks & $\begin{array}{l}\text { Exp: Platelet gel (autologe } \mathrm{l}^{\oplus} \text { ) versus } \\
\text { CTR: Placebo gel }\end{array}$ & $\begin{array}{l}\text { Proportion of healed ulcers and } \\
\text { time to healing } 24 \text { weeks }\end{array}$ & $\begin{array}{c}\text { Exp: } 13 / 16 \\
\text { CTR: } 8 / 19 \\
\text { Time to healing shorter in Exp group } \\
(p=0.018) 12 \text { week treatment phase } \\
\text { Safety evaluation }\end{array}$ \\
\hline Jeong (2010) [27] & $\begin{array}{c}\text { RCT } \\
\text { Exp } N=52 \text { patients } \\
\text { CTR } N=48 \text { patients }\end{array}$ & DFU & $>4$ weeks & $\begin{array}{c}\text { Exp: Blood Bank Platelet } \\
\text { Concentrate versus } \\
\text { CTR: treatment with topical fibrinogen } \\
\text { and thrombin } \\
\end{array}$ & $\begin{array}{l}\text { Complete wound healing was } \\
\text { achieved/12 weeks }\end{array}$ & $\begin{array}{c}\text { Exp: } 79 \% \\
\text { CTR: } 46 \%(p<0.05)\end{array}$ \\
\hline Kakagia (2007) [28] & $\begin{aligned} R C T \\
\text { Exp } A N=17 \text { patients } \\
\text { Exp } B N=17 \text { patients } \\
\text { Exp } C N=17 \text { patients } \\
\end{aligned}$ & $\begin{array}{l}51 \text { patients with } \\
\text { significant tissue } \\
\text { defects of the foot }\end{array}$ & $>3$ months & $\begin{array}{c}\text { Exp A: oxidized cellulose/collagen } \\
\text { Exp B: autologous PRP } \\
\text { Exp C: a combination of both }\end{array}$ & $\begin{array}{l}\text { Ulcer dimension within } 8 \text { week } \\
\text { follow-up }\end{array}$ & No differences between groups \\
\hline Karimi (2015) [29] & $\begin{array}{c}\text { RCT } \\
\text { Exp } N=25 \text { patients } \\
\text { CTR } N=25 \text { patients } \\
\end{array}$ & DFU & No limit & $\begin{array}{c}\text { Exp: PRP } \\
\text { CTRL: conventional management }\end{array}$ & Ulcer's depth in three weeks & $\begin{array}{l}\text { Exp: } 4.56 \pm 5.76 \\
\text { CTRL: } 13.03 \pm 14.1 p=0.004\end{array}$ \\
\hline Krupski (1991) [31] & $\begin{array}{c}\text { RCT } \\
\text { Exp } N=10 \text { patients } \\
\text { CTR } N=8 \text { patients }\end{array}$ & $\begin{array}{l}\text { Number ulcers: } 26 \\
\text { Wound aetiology: } \\
\text { Mixed } 78 \% \text { diabetic, } \\
72 \% \text { occlusive } \\
\text { peripheral vascular } \\
\text { disease, and } 28 \% \\
\text { venous disease }\end{array}$ & $>8$ weeks & $\begin{array}{l}\text { Exp: PDWHF topical solution) } \\
\text { every } 12 \mathrm{~h} \\
\text { CTR: saline solution every } 12 \mathrm{~h} \\
\text { The treatment is applied by the patient }\end{array}$ & Total epithelialization/12 weeks & $\begin{array}{c}\text { Exp: } 4 / 17 \\
\text { CTR: } 3 / 9 \\
\text { Healing rate Exp: }-4.3(12.2) \\
\mathrm{cm}^{2} / \text { week } \\
\text { CTR: } 1.9(2.7) \mathrm{cm}^{2} / \text { week }\end{array}$ \\
\hline Li L (2015) [32] & $\begin{array}{c}\text { RCT } \\
\text { Exp } N=59 \text { patients } \\
\text { CTR } N=58 \text { patients }\end{array}$ & DFU refractory & $>2$ weeks & $\begin{array}{l}\text { Autologous platelet-rich gel, double } \\
\text { spinning and calcium } \\
\text { gluconate activation } \\
\text { Repeated PR-gel application if reduction } \\
\text { of wound area did not reach } 80 \% \\
\text { reduction } 2 \text { weeks after treatment }\end{array}$ & $\begin{array}{l}\text { Reduction rate at the end of week } \\
12 \text { th/12 weeks } \\
\text { Lost to follow-up Exp: 6, CTR: } 5\end{array}$ & $\begin{array}{l}\text { Healing velocity faster in PR-gel } \\
\text { group, } p=0.020\end{array}$ \\
\hline
\end{tabular}


Table 2. Cont.

\begin{tabular}{|c|c|c|c|c|c|c|}
\hline $\begin{array}{l}\text { Author (Year) } \\
\text { [Reference] }\end{array}$ & $\begin{array}{l}\text { Study Design } \\
\text { Experimental/ } \\
\text { Control Group }\end{array}$ & Patient Population & Chronicity of the Ulcer & $\begin{array}{l}\text { Biological Intervention and Control } \\
\text { Management }\end{array}$ & Outcomes/Follow-Up & Differences and Statistical Results \\
\hline Moneib (2017) [33] & $\begin{array}{c}\text { CT } \\
\operatorname{Exp} N=20 \\
\text { CTR } N=20\end{array}$ & $\begin{array}{l}\text { Venous leg ulcer } \\
\text { Ankle/brachial } \\
\text { index }>0.80\end{array}$ & $>6$ months & $\begin{array}{l}\text { PR-gel double spinning activation with } \\
\text { calcium gluconate + compression } \\
\text { CTR: saline management + compression }\end{array}$ & $\begin{array}{l}\text { Reduction in ulcer size expressed } \\
\text { as percentage improvement in area }\end{array}$ & $\begin{array}{l}\text { Higher reduction in ulcer size in PRP } \\
\text { group compared with control } \\
p<0.0001\end{array}$ \\
\hline Obolensky (2017) [34] & $\begin{array}{c}\text { CT } \\
\text { Exp } N=50 \text { patients } \\
\text { CTR } N=50 \text { patients }\end{array}$ & $\begin{array}{l}\text { Non-healing wounds } \\
\text { of different etiology, } \\
82 \% \text { of the wounds } \\
\text { located in lower limb }\end{array}$ & $>6$ weeks & $\begin{array}{l}\text { Exp: Pure PRP (single spinning) } \\
\text { CTR: conventional management }\end{array}$ & $\begin{array}{l}\text { Epithelialization time } \\
\text { Hospitalization time } \\
\text { Economic effect }\end{array}$ & $\begin{array}{l}\text { Epithelialization: Exp: } 42.3 \text { days } \\
\text { (SD: } 5.7 \text { ) } \\
\text { CTR: 123.8 days (SD: } 25.3 \text { ) } \\
\text { Hospitalization Exp } 8.4 \text { days } \\
\text { (SD: } 1.5 \text { ) CRR: } 18.1 \text { (SD: } 1.6 \text { ) } \\
\text { €736.81 in savings per patient } \\
\text { PRP group }\end{array}$ \\
\hline Pravin (2016) [35] & $\begin{array}{c}\text { RCT } \\
\text { Exp1 } N=16 \\
\operatorname{Exp} 2 N=15\end{array}$ & $\begin{array}{l}22 \text { venous ulcers, } 1 \\
\text { vasculitis, } 1 \text { traumatic, } \\
2 \text { diabetic, } 4 \\
\text { trophic ulcers }\end{array}$ & $>8$ weeks & $\begin{array}{l}\text { Exp1: PRP (double spinning) } \\
\text { Exp2: L-PRF (single spinning) } \\
\text { Weekly administration for } 6 \text { weeks }\end{array}$ & $\begin{array}{c}\text { Study period } 6 \text { weeks, follow-up } 6 \\
\text { weeks }\end{array}$ & $\begin{array}{l}\text { Mean duration of healing: } 5.7 \text { weeks } \\
\text { in L-PRF and } 6.5 \text { weeks in PRP } \\
\qquad p=0.034\end{array}$ \\
\hline Saad Setta (2011) [36] & $\begin{array}{c}\text { RCT } \\
\text { Exp } N=12 \text { patients } \\
\text { CTR } N=12 \text { patients }\end{array}$ & Non healing DFUs & $>3$ months & $\begin{array}{l}\text { Exp: gelified platelet-rich plasma (with } \\
\text { bovine thrombin and } \mathrm{CaCl}_{2} \text { ) } \\
\text { CTR: platelet-poor plasma Treatment } \\
\text { applied twice weekly until closure } \\
\text { (maximum } 20 \text { weeks) }\end{array}$ & $\begin{array}{l}\text { Healing duration in weeks/ } \\
20 \text { weeks } \\
\text { Lost to follow-up: } 3\end{array}$ & $\begin{array}{l}\text { Exp: } 11.5 \text { weeks } \\
\text { CTR: } 17.1 \text { weeks, } p<0.005\end{array}$ \\
\hline $\begin{array}{l}\text { Saldalamachia } \\
\text { (2004) [37] }\end{array}$ & $\begin{array}{c}\text { CT } \\
\text { Exp } N=7 \text { patients } \\
\text { CTR } N=7 \text { patients }\end{array}$ & $\begin{array}{l}\text { Diabetic foot } \\
15 \text { patients }\end{array}$ & $>8$ weeks & $\begin{array}{c}\text { Exp: autologous gelified PRP, topical } \\
\text { application } \\
\text { CTR: standard care Weekly application } \\
\text { for } 5 \text { weeks } \\
\text { No description of PRP product }\end{array}$ & $\begin{array}{c}\text { Reduction rate }=[(\text { final area } \\
\left(\mathrm{mm}^{2}\right)-\text { nitial area }\left(\mathrm{mm}^{2}\right) / \text { initial } \\
\left.\text { area }\left(\mathrm{mm}^{2}\right)\right] / 5 \text { weeks } \\
\text { Lost to follow-up: } 1\end{array}$ & $\begin{array}{l}\text { Reduction rate } 71.9(22.5) \text { vs. } 9.2 \\
\quad(67.8) p<0.039 \\
\text { or reduction of } 50 \% \text { or more was } \\
\text { Exp: } 71 \% \text { and CTR: } 29 \%\end{array}$ \\
\hline Sennet (2003) [38] & $\begin{array}{c}\text { RCT } \\
\text { Exp } N=8 \text { patients } \\
\text { CTR } N=7 \text { patients }\end{array}$ & $\begin{array}{l}\text { Chronic venous leg } \\
\text { ulcer }\end{array}$ & $>2$ months & $\begin{array}{l}\text { Exp: frozen platelet lysate obtained by } \\
\text { sonication } 10^{7} \mathrm{plt} / \mathrm{cm}^{2} \text { in saline } \\
\text { CTR: saline }\end{array}$ & $\begin{array}{l}\text { Mean percent reduction in ulcer } \\
\text { area/12 weeks }\end{array}$ & $\begin{array}{c}\text { Exp: } 26.2 \% \text { CTR: } 15.2 \% \\
\quad(p=0.94) .\end{array}$ \\
\hline Stacey (2000) [39] & $\begin{array}{c}\text { RCT } \\
\text { Exp } N=42 \text { patients } \\
\text { CTR } N=44 \text { patients } \\
\end{array}$ & $\begin{array}{l}\text { Venous ulceration, } \\
\text { with no other possible } \\
\text { cause for poor healing }\end{array}$ & $>3$ months & $\begin{array}{c}\text { Exp: autologous platelet lysate (without } \\
\text { plasma) } 2 \times 10^{9} \mathrm{plt} / \mathrm{mL} \\
\text { CTR: PBS }\end{array}$ & $\begin{array}{l}\text { Ulcer healing/9 months } \\
\text { Lost to follow-up: } 11\end{array}$ & $\begin{array}{c}\text { No significant differences } \\
\text { between treatment } \\
\text { Only ulcer size influenced healing } \\
\end{array}$ \\
\hline Steed (1992) [40] & $\begin{array}{c}\text { RCT } \\
\text { Exp } N=7 \text { patients } \\
\text { CTR } N=6 \text { patients }\end{array}$ & $\begin{array}{l}13 \text { subjects with } \\
\text { neurotrophic ulcer }\end{array}$ & $>8$ weeks & $\begin{array}{l}\text { Exp: PDWHF (obtained from washed } \\
\text { allogeneic platelets (without plasma) } \\
\text { stimulated with thrombin } \\
\text { CTR: placebo }\end{array}$ & $\begin{array}{l}\text { Ulcer healing/Followed for } \\
20 \text { weeks }\end{array}$ & $\begin{array}{l}\text { Exp: } 5 / 7 \text { ulcers healed by week } 15 \text { th } \\
\text { CTR: } 1 / 6 \text { ulcers healed by week } 20 \text { th }\end{array}$ \\
\hline
\end{tabular}

Abbreviations: $\mathrm{CT}=$ controlled trial; $\mathrm{CTR}=$ control group; $\mathrm{DFU}=$ diabetic foot ulcer; Exp $=$ Experimental treatment; $\mathrm{L}-\mathrm{PRF}=$ leukocyte-rich platelet-rich fibrin; PDWHF $=$ platelet derived wound healing formula (or factors); PR (gel) = platelet rich; PRP = platelet rich plasma; PTA = percutaneous transluminal angioplasty; $\mathrm{RCT}=$ randomised controlled trial; $\mathrm{SD}=$ standard deviation. 


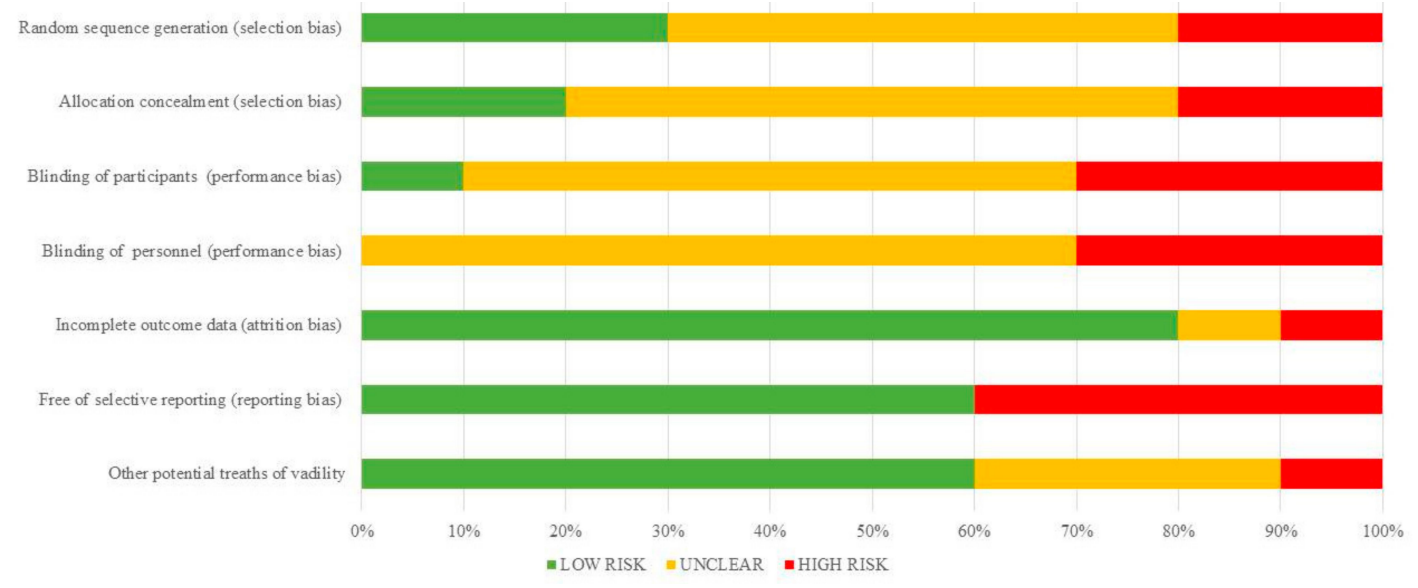

Figure 1. Quantification synthesis of risk of bias (cell therapies).

\subsection{Cell-Based Studies}

Ten controlled studies involving MSC derived therapies were identified, with a total of 261 patients in the experimental arm and 219 patients in the control group [13-22]. Studies were published between 2005 and 2017. Most studies included DFU [13-20], and one study included ulcers from different etiology (post-trauma, diabetic, arterial, and venous) [21].

There were eight randomized controlled trials (RCTs) [13,15-18,20-22] and two controlled studies [14,19]. The number of patients per experimental group ranged from 10 to 42 . Standard wound care or placebo were used as control in most studies. The experimental products tested were: autologous BMC injections [14,16-18,20,22], ex vivo expanded bone marrow mesenchymal stromal cells (BM-MSCs) $[13,17,18]$, SVF $[15,19,21]$, and peripheral blood stem cells (PBMNC) after granulocyte colony-stimulating factor (G-CSF) mobilization [14]. Two studies compared two cell products, BMC versus PBMNC [14] and BMC versus BM-MSC enriched in CD90+ cells [17]. Whether ex vivo expanded BM-MSCs were superior to BMC was explored in one three armed study [18].

There were no differences between BMC and PBMNCs [14]. When BM-MSCs and BMC were compared [18], the former showed better outcomes, but both cell treatments were better than saline. Two other studies, involving DFU, used SVF combined with fibrinogen/fibrin [15] and with PRP [21].

The patient population was predominantly formed by diabetic patients (in some cases associated with critical limb ischemia [14,18,20]. Main outcome measurements included parameters related to ulcer healing and the rate of amputations. Cell therapies enhanced outcomes in all studies (Table 1).

The risk of bias of cell therapy studies are shown in Table 3 and summarized in Figure 1. The risk of bias arising from the method of generation allocation sequence was considered as low in three trials $[15,16,18]$, five were unclear [13,17,20-22], and the two remaining had a high risk of bias [14,19]. The risk of bias arising from the method of allocation concealed was considered low in two trials $[13,16]$. But in other six studies this was not specified [15,17,18,20-22] and two studies did not use adequate allocation concealment $[14,19]$ (Table 3). Concerning performance bias, insufficient description of the blinding procedure was found in six studies $[13,16,17,20-22]$. The remaining three studies had high risk of bias $[14,15,19]$. The risk of attrition bias was rated as low in seven trials $[13-17,20]$, and was unclear in three studies $[18,19,21]$. Reporting bias was rated as low in four trials $[13,16,17,19]$. 
Table 3. Risk of bias summary for cell therapies studies.

\begin{tabular}{|c|c|c|c|c|c|c|c|c|c|c|}
\hline Sources of Bias & $\begin{array}{c}\text { Dash } \\
(2009)[13]\end{array}$ & $\begin{array}{c}\text { Dubsky } \\
(2013)[14]\end{array}$ & $\begin{array}{c}\text { Han } \\
\text { (2009) [15] }\end{array}$ & $\begin{array}{c}\text { Jain } \\
\text { (2011) [16] }\end{array}$ & $\begin{array}{c}\text { Kirana } \\
(2012)[17]\end{array}$ & $\begin{array}{c}\text { Lu } \\
\text { (2011) [18] }\end{array}$ & $\begin{array}{c}\text { Marino } \\
\text { (2013) [19] }\end{array}$ & $\begin{array}{l}\text { Procházka } \\
\text { (2010) [20] }\end{array}$ & $\begin{array}{c}\text { Raposio } \\
\text { (2016) [21] }\end{array}$ & $\begin{array}{c}\text { Walter } \\
\text { (2011) [22] }\end{array}$ \\
\hline $\begin{array}{l}\text { Random sequence generation } \\
\text { (selection bias) }\end{array}$ & ? & - & + & + & ? & + & - & ? & ? & ? \\
\hline $\begin{array}{l}\text { Allocation concealment } \\
\text { (selection bias) }\end{array}$ & + & - & ? & + & ? & $?$ & - & ? & ? & ? \\
\hline $\begin{array}{l}\text { Blinding of patients } \\
\text { (performance bias) }\end{array}$ & $?$ & - & - & $?$ & $?$ & + & - & $?$ & $?$ & $?$ \\
\hline $\begin{array}{l}\text { Blinding of personnel } \\
\text { (performance bias) }\end{array}$ & $?$ & - & - & $?$ & $?$ & $?$ & - & $?$ & $?$ & $?$ \\
\hline $\begin{array}{l}\text { Incomplete outcome data } \\
\text { (attrition bias) }\end{array}$ & + & + & + & + & + & + & $?$ & + & + & - \\
\hline Selective reporting (reporting bias) & - & + & + & - & - & + & - & + & + & + \\
\hline Other bias & + & + & ? & + & ? & + & ? & + & - & + \\
\hline
\end{tabular}

+: low risk of bias; -: high risk of bias; ?: unclear risk of bias.

\subsection{Platelet-Based Therapies}

We identified 18 studies involving the use of platelets or PRP in chronic leg ulcers; they were published between 1986 and 2017 [23-40]. A summary of all the studies is shown in Table 2.

The number of participants per group ranged between seven and 59. The number of participants in the experimental and control groups were 408 and 384, respectively. All of them were RCT except three $[33,34,40]$, but most studies were underpowered [24,25,28,30,31,36-38]. All of the studies except one [28] were two armed. The biological interventions were highly variable, not only concerning the composition of platelet products, but also the number of applications, and the interval between applications. Topical PRP gel was used in seven studies [23-26,32,36,37]. In these studies PRP was stimulated with thrombin and/or $\mathrm{Ca}_{2} \mathrm{Cl}$ or calcium gluconate to initiate coagulation and platelet activation in order to obtain platelt rich (PR) gels. The presence of leukocytes in PRPs and the platelet enrichment relative to peripheral blood are hardly described. One study [35] compared PRP that was obtained through double spinning with leukocyte-platelet rich fibrin (L-PRF) that was obtained through single spinning, and found better outcomes in L-PRP treated patients. PDWHF (platelet derived wound healing factors, i.e., platelet secretome) was topically applied in three studies $[30,31,40]$. Two studies $[27,40]$ used allogeneic platelets. Platelet lysate obtained by freeze/thawing or sonication was used in two studies [38,39]. Knighton et al. used PDWHF mixed with crystalline collagen [30]. The frequency of application varied between twice daily [30], twice weekly [23,36], or weekly [35].

Time to healing or reduction in the ulcer area was the most common outcome measurements. Seven trials involved predominantly diabetic patients [23,26,29,31,32,36,37], while mixed ulcer etiology were involved in the other studies. Outcome results favored experimental treatments in eleven studies $[23,24,26-28,30-32,36,37,40]$.

The risk of bias for individual studies is shown in Table 4 and the summary is depicted in Figure 2. The risk of bias arising from the method of generation allocation sequence was considered low in eight trials $[24-28,31,32,39]$, and six of these trials had low risk as regards the method of concealed allocation [25,26,30-32,39]. Three had a high risk of selection bias [33,36,37]. Overall, most of the studies had a high or unclear risk of performance bias, except for one study [31]. The risk of attrition bias was rated high in three studies [24,26,30], and reporting bias was high in seven studies $[28,30,33,35,37,38,40]$. 
Table 4. Risk of bias summary for PRP studies.

\begin{tabular}{|c|c|c|c|c|c|c|c|c|c|c|c|c|c|c|c|c|c|c|}
\hline Sources of bias & $\begin{array}{c}\text { Ahmed } \\
(2017) \\
{[23]}\end{array}$ & $\begin{array}{c}\text { Anitua } \\
(2008) \\
{[24]}\end{array}$ & $\begin{array}{c}\text { Danielsen } \\
(2008) \\
{[25]}\end{array}$ & $\begin{array}{c}\text { Driver } \\
(2006) \\
{[26]} \\
\end{array}$ & $\begin{array}{c}\text { Jeong } \\
\text { (2010) } \\
\text { [27] }\end{array}$ & $\begin{array}{c}\text { Kakagia } \\
(2007) \\
{[28]}\end{array}$ & $\begin{array}{c}\text { Karimi } \\
(2015) \\
{[29]} \\
\end{array}$ & $\begin{array}{c}\text { Knighton } \\
\text { (1990) } \\
{[30]}\end{array}$ & $\begin{array}{l}\text { Krupski } \\
\text { (1991) } \\
{[31]}\end{array}$ & $\begin{array}{c}\mathrm{Li} \\
(2015) \\
{[32]}\end{array}$ & $\begin{array}{c}\text { Moneib } \\
\text { (2017) } \\
{[33][}\end{array}$ & $\begin{array}{l}\text { Obolenski } \\
\text { (2017) [34] }\end{array}$ & $\begin{array}{c}\text { Pravin } \\
(2016) \\
{[35]} \\
\end{array}$ & $\begin{array}{l}\text { Saad Setta } \\
(2011)[36]\end{array}$ & $\begin{array}{l}\text { Saldalamacchia } \\
\quad(2004)[37]\end{array}$ & $\begin{array}{c}\text { Senet } \\
(2003) \\
{[38]} \\
\end{array}$ & $\begin{array}{c}\text { Stacey } \\
(2000) \\
{[39]}\end{array}$ & $\begin{array}{c}\text { Steed } \\
(1992) \\
{[40]}\end{array}$ \\
\hline $\begin{array}{l}\text { Random sequence generation } \\
\text { (selection bias) }\end{array}$ & $?$ & + & + & + & + & + & ? & ? & + & + & - & ? & ? & - & - & $?$ & + & $?$ \\
\hline $\begin{array}{l}\text { Allocation concealment } \\
\text { (selection bias) }\end{array}$ & $?$ & ? & + & + & ? & $?$ & $?$ & + & + & + & - & $?$ & $?$ & - & - & $?$ & + & $?$ \\
\hline $\begin{array}{l}\text { Blinding of patients } \\
\text { (performance bias) }\end{array}$ & ? & - & ? & + & ? & ? & ? & + & + & ? & - & ? & - & - & - & ? & ? & $?$ \\
\hline $\begin{array}{l}\text { Blinding of personnel } \\
\text { (performance bias) }\end{array}$ & $?$ & - & ? & - & ? & ? & ? & - & + & ? & - & ? & - & - & - & ? & ? & $?$ \\
\hline $\begin{array}{l}\text { Incomplete outcome data } \\
\text { (attrition bias) }\end{array}$ & ? & - & + & - & ? & + & + & - & + & + & + & + & ? & + & + & + & + & + \\
\hline $\begin{array}{l}\text { Selective reporting } \\
\text { (reporting bias) }\end{array}$ & + & + & $?$ & + & + & - & + & - & + & + & - & $?$ & - & + & - & - & + & - \\
\hline Other bias & $?$ & - & + & $?$ & $?$ & $?$ & + & - & + & + & $?$ & $?$ & $?$ & + & ? & + & + & - \\
\hline
\end{tabular}

+: low risk of bias; -: high risk of bias; ?: unclear risk of bias.

PLATELET THERAPIES

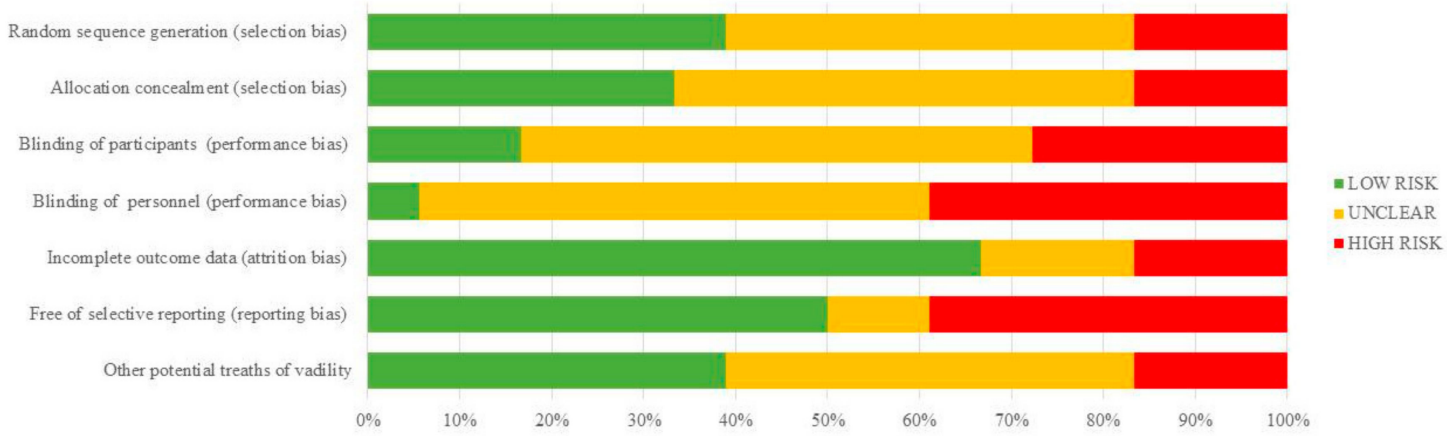

Figure 2. Quantification synthesis of risk of bias (platelet therapies). 


\section{Discussion}

We have reviewed controlled studies examining the efficacy of biological adjuvants, mainly cellular products (i.e., BMC and SVF) and platelet-derived products for chronic leg ulcer management. We identified ten controlled studies using mesenchymal-stromal cell based therapies, and 18 studies examining platelet derived products that were applied locally to augment wound healing. Overall, a general positive effect on ulcer size reduction was found in favor of these biological interventions.

Generally, adjuvants are applied when there is a lack of adequate progress on healing. Crucially, DFU precedes $85 \%$ of all lower limb amputations [41], thus the importance of developing efficient adjuvant treatments for rapid healing when the ulcer outcome is stalled. As shown in Tables 1 and 2 summarizing our findings, most patients were diabetic, emphasizing the unmet medical need of this patient population.

Ulcers in the lower extremity may develop from a diversity of conditions, including neuropathy, venous hypertension, mechanical pressure, and ischemia. The latter has been treated with intramuscular injections of either bone marrow derived products, or PBMNCs after mobilization with G-CSF. After systematic review and meta-analysis, data revealed that bone marrow products, but not G-CSF mobilized PBMNCs, improved the surrogate indexes of ischemia [42,43].

There are different strategies to deliver MSCs, from injectable mixtures of cell populations, as is the case with BMC, and SVF, to refined MSC preparations. Most of the studies included in this review used BMC (five studies) or SVF (three studies), which can be prepared at the point of care, using automated closed systems, single use consumables, and clinical grade reagents. These products contain mixed cell phenotypes, in different degree of maturation, including mature cells (adipocytes, fibroblasts, smooth muscle cells, endothelial blood cells and macrophages), progenitors (pre-adipocytes and endothelial, vascular, and hematopoietic progenitors) and stem cells, including MSCs, hematopoietic stem cells, pericytes and supra-adventitial cells [44-46]. The rationale for their application is that the non-healing wound is deficient in cells and healing proteins. The main mechanism of action of MSCs consists on paracrine interactions with other cell populations, thereby providing a sustained healing factor delivery to cope with tissue needs [47]. The main mechanisms of action of the mixed cell populations that form part of BMC and SVF have not been described yet.

An advantage of these cellular products is that medical devices and protocols are commercialized to prepare these products (BMC and SVF) at the point of care, facilitating rapid implementation if they were effective. However, our results highlight the low number of treated patients (292), and the great heterogeneity regarding not only cell products, but also outcome measurements, and poor assessment of the power of the study to discriminate the effect sizes of the outcomes; in fact, sample size calculation for at least one clinically important effect is seldom reported [18,22].

Importantly, and relevant to the use of these biological adjuncts for healing, is the fact that no worrying safety concerns were reported in relation to intramuscular injections of this type of therapy [42]. Moreover, the safety of these products in other medical areas, such as intraarticular administration in osteoarthritis, has been ascertained [48-50].

Regenerative Medicine treatments are not restricted to cell therapies. There is another category of molecular products obtained from peripheral blood, i.e., PRPs and other platelet therapies. PRP research has increased spectacularly in the last decade. In the 60s, hematologists used PRPs, as a transfusion product for patients with coagulation problems. But, these treatments breathe new life when they were used in the treatment of chronic leg ulcers [51]. These were the first clinical applications of platelets outside of the blood stream, and were followed by the use of PRPs in other medical areas, especially in the field of orthopedics and sports medicine [52,53]. Since then, PRP research has explored the molecular interactions of platelets and plasma secretome with different cell phenotypes [54], thereby constituting a subdiscipline of regenerative medicine. Basic science indicates that PRPs may be promising in the field of wound care, because it enhances cell migration, proliferation, angiogenesis, and tissue anabolism [55]. However, research in novel therapies takes time to develop and optimal indications and protocols are lacking. As we can see in this review, 
there are different procedures for PRP or PDWHF application (topically or injected), different platelet and leukocyte concentrations, and combination products (i.e., (PRP + collagen powder), (PRP + oxidized cellulose-collagen)). Most studies [23-26,32,33,36,37] used coagulated PRP, so-called PR gel. These products contain the platelet secretome along with plasma proteins, but activated platelets on their own (without plasma) are also effective [30,31,40].

There are two recent reviews on platelet therapies for wound healing [56,57]. Carter et al. [56] have reviewed and metaanalysed the use of PR-gel on wound healing, acute, and chronic conditions, including prospective and retrospective studies that are published in journals and in congress. The review included 21 studies and nine studies were included in the metaanalysis. The results indicated that PRPs favored healing in chronic ulcers and that the presence of infection was reduced in acute wounds treated with PRP [56]. Martinez-Zapata et al. [57] included 10 RCTs in chronic wounds in their metaanalysis. Three of these RCTs involved DFU and three studies involved venous leg ulcers. Overall analysis did not shed light on PRP effectiveness, but the results indicated that autologous PRP can enhance DFU healing when compared with standard care, although the value of the evidence is low.

Insufficient description of the biological intervention is a major drawback in published studies. At present, the need of minimum reporting standards for biological therapies is recognized [58], and to advance in the field, a consensus regarding minimal requirements for reporting on biological products is obligatory.

PRP helps healing by releasing a physiological pool of proteins involved in different biological processes, including angiogenesis, ECM synthesis, and remodeling. It consists of more than 300 proteins that are released from activated platelets within a fibrin scaffold formed upon plasma coagulation. Alternatively, platelets are used without fibrin. Regarding platelet concentration, PRP containing a near-physiological concentration of platelets (Autologel System, Cytomedix, Gaithersburg, MD, USA) induced a healing response in $96.5 \%$ of wounds within 2.2 weeks with 2.8 treatments, in a large observational study using a multicenter registry database ( 39 centers). The authors concluded that PRP gel could trigger the healing process as positive changes were assessed in 275 of 285 wounds $[59,60]$. Furthermore, registry data of 26,599 patients, treated between 1988 and 1997 in various Wound Care Centers that are associated with Curative Health Services, showed that platelet releasate was more effective than the standard care, especially in the most severe wounds affecting deeper anatomical structures [61].

Different healing impediments can have varying leverage depending on the specific patient, emphasizing the need to embrace personalized medicine approaches [62]. Healing can be hindered by infection, and osteomyelitis is common in DFU. Sixty-four patients with chronic osteomyelitis randomized to artificial bone implantation with or without autologous PRP and bone marrow implantation showed enhanced bone regeneration in the group with the biological intervention [63].

Importantly, the most suitable dressing to maintain cell viability and optimize the interactions of healing proteins with the ulcer bed has to be defined cautiously [64]. Likewise, needling the fibrotic tissue, while injecting PRP, can eliminate the fibrotic barrier around the ulcer bed, thereby improving healing in selected patients [65].

Based on the current medical literature, it is unclear when these biological adjuvants should be considered, and their place in the decision tree when other treatments are not effective. The need of high quality clinical research is reflected in our review. Although results may be promising, comprehensive reporting that included a clear description of treatment protocols (a description of dressings), providing the composition of the biological product and clinical outcomes, including ulcer size reduction, the time to heal, and the rate of healing are mandatory. Likewise, conducting clinical trials with high quality methodology can help to provide evidence for better clinical care. 


\section{Materials and Methods}

We followed the recommendations of the Cochrane Handbook for Systematic Reviews [66] and performed the study according to PRISMA (Preferred Reporting Items for Systematic Reviews and Meta-analysis) statement [67].

\subsection{Search Strategy}

We did a comprehensive systematic search in MEDLINE [68], EMBASE [69], and The Cochrane Library Clinical Trials Database [70] until week 30 July 2017. The search included human clinical studies, written in English. The following algorithm was used to search in MEDLINE via PubMed: (skin ulcer\$ OR foot ulcer\$ OR diabetic foot OR diabetic feet OR leg ulcer\$ OR varicose ulcer\$ OR venous ulcer\$ OR stasis ulcer\$ OR arterial ulcer\$ OR neuropathic ulcer\$ OR chronic ulcer OR chronic wound) AND (cell OR platelet-derived wound healing factor OR PDWHF OR platelet-rich OR (platelet adj rich) OR "platelet rich plasma" OR "platelet-rich plasma" OR PRP OR "platelet gel\$"). We searched through the other data bases with a similar strategy, by using a combination of the terms: "platelet rich plasma", "skin leg ulcer" and "cell therapy". We manually checked the references of selected articles to identify additional eligible studies.

\subsection{Selection Criteria}

Two reviewers independently assessed each title and abstract of all the articles, and selected a manuscript according to the following criteria: all clinical trials, randomised, and non-randomised comparative cohorts that provided scientific evidence on the efficacy of biological interventions in lower limb ulcers versus other therapies or conventional management were eligible for inclusion. Retrospective observational studies were not included. The experimental treatment had to be any biological adjuvant agent, including mesenchymal stromal cells-based products, i.e., adipose or BMR or MSCs, as biological adjuvants applied locally to the ulcer bed or injected into the edges of the ulcer were included. Studies involving intramuscular injections of mobilized peripheral blood cells or bone marrow derived cells to treat patients with critical limb ischemia (with and without ulcers) were excluded. In addition, we included studies using biological adjuvant agents, such as PRP or PDWHF, (but not recombinant growth factor therapies). Three authors reviewed separately the final list of eligible studies and reach a consensus regarding controversies. (Figure 3).

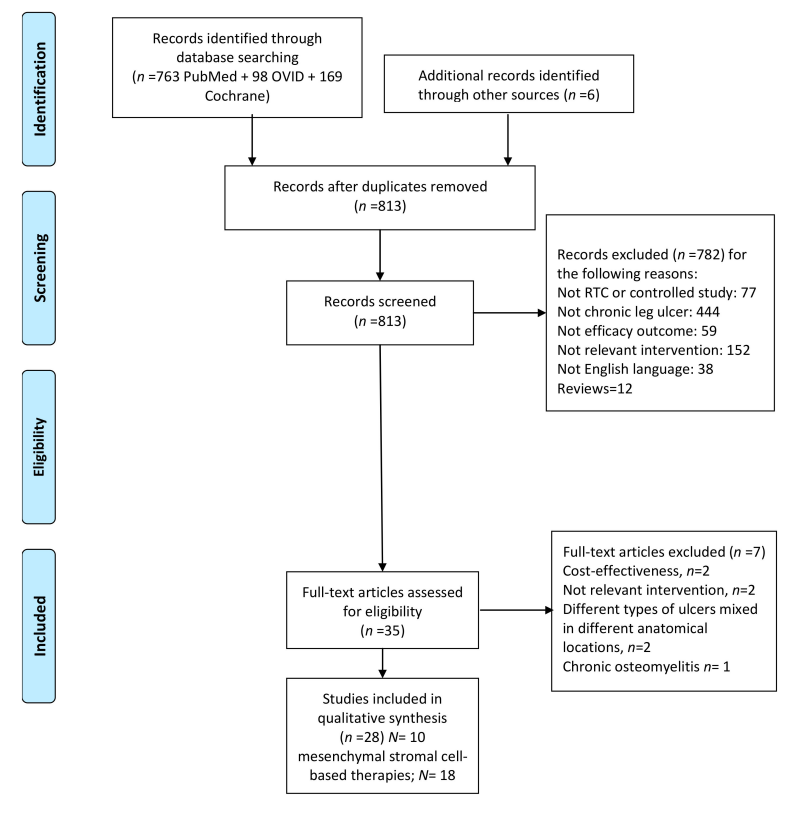

Figure 3. Flow diagram of study selection. 
Results are displayed in two independent Tables: Table 1 summarizes cells' studies and Table 2 studies involving platelet derived products.

\subsection{Data Extraction}

Full texts were acquired for all of the studies matching inclusion criteria. The following data, related to ulcer healing, were extracted: healing rate, time required for complete wound healing, decrease in the wound area, ulcer size or rate of major amputation relative to the control group. Other extracted data included patient population and pathology, the number of patients in each group, ulcer chronicity, description of the intervention, and control management.

\subsection{Risk of Bias Assessment}

Studies were assessed for quality following the Cochrane Risk of Bias Tool [66].

Two authors independently evaluated the risk of bias of the selected cell intervention studies and two different authors assessed the studies involving platelet therapies, according to Cochrane guidelines.

Acknowledgments: This work was supported in part by the Department of Health and Consumer Affairs of the Government of the Basque Country (Grant No. 2011111127), Osakidetza, and Basque Health Service.

Author Contributions: Isabel Andia and Natalia Burgos-Alonso conception and drafting of the article; Natalia Burgos-Alonso, Igone Lobato, and Igone Hernández did a comprehensive systematic search in databases and manually checked the references of selected articles to identify additional eligible studies; Natalia Burgos-Alonso and Isabel Andia independently assessed each title and abstract of all the articles and selected the articles that were confirmed by Begoña Rodríguez. Kepa San Sebastian and Igone Lobato independently assessed the risk of bias, which was confirmed by Gontzal Grandes. All authors read and approved the manuscript.

Conflicts of Interest: The authors declare no conflict of interest.

\section{References}

1. Driver, V.R.; Fabbi, M.; Lavery, L.A.; Gibbons, G. The costs of diabetic foot: The economic case for the limb salvage team. J. Vasc. Surg. 2010, 52, 17s-22s. [CrossRef] [PubMed]

2. Fife, C.E.; Carter, M.J. Wound care outcomes and associated cost among patients treated in us outpatient wound centers: Data from the US wound registry. Wounds Compend. Clin. Res. Pract. 2012, 24, 10-17.

3. Global Industry Analysis, I. Advances Wound Care-A Global Strategies Bussines Report. Available online: http:/ / www.strategyr.com/Advanced_Wound_Care_Market_Report.asp\#sthash.FTA2Umdo.mqm11Rio. dpbs (accessed on 4 October 2017).

4. Fife, C.; Walker, D.; Thomson, B.; Carter, M. Limitations of daily living activities in patients with venous stasis ulcers undergoing compression bandaging: Problems with the concept of self-bandaging. Wounds Compend. Clin. Res. Pract. 2007, 19, 255-257.

5. Sen, C.K. Wound healing essentials: Let there be oxygen. Wound Repair Regen. 2009, 17, 1-18. [CrossRef] [PubMed]

6. Rogers, L.C.; Bevilacqua, N.J.; Armstrong, D.G. The use of marrow-derived stem cells to accelerate healing in chronic wounds. Int. Wound J. 2008, 5, 20-25. [CrossRef] [PubMed]

7. Carter, M.J.; Waycaster, C.; Schaum, K.; Gilligan, A.M. Cost-effectiveness of three adjunct cellular/tissue-derived products used in the management of chronic venous leg ulcers. Value Health 2014, 17, 801-813. [CrossRef] [PubMed]

8. Fernandez-Montequin, J.I.; Valenzuela-Silva, C.M.; Diaz, O.G.; Savigne, W.; Sancho-Soutelo, N.; Rivero-Fernandez, F.; Sanchez-Penton, P.; Morejon-Vega, L.; Artaza-Sanz, H.; Garcia-Herrera, A.; et al. Intra-lesional injections of recombinant human epidermal growth factor promote granulation and healing in advanced diabetic foot ulcers: Multicenter, randomised, placebo-controlled, double-blind study. Int. Wound J. 2009, 6, 432-443. [CrossRef] [PubMed] 
9. Yonemitsu, Y.; Matsumoto, T.; Itoh, H.; Okazaki, J.; Uchiyama, M.; Yoshida, K.; Onimaru, M.; Onohara, T.; Inoguchi, H.; Kyuragi, R.; et al. DVC1-0101 to treat peripheral arterial disease: A phase I/IIa open-label dose-escalation clinical trial. Mol. Ther. J. Am. Soc. Gene Ther. 2013, 21,707-714. [CrossRef] [PubMed]

10. Senet, P.; Vicaut, E.; Beneton, N.; Debure, C.; Lok, C.; Chosidow, O. Topical treatment of hypertensive leg ulcers with platelet-derived growth factor-bb: A randomized controlled trial. Arch. Dermatol. 2011, 147, 926-930. [CrossRef] [PubMed]

11. Andia, I.; Abate, M. Platelet-rich plasma: Underlying biology and clinical correlates. Regen. Med. 2013, 8, 645-658. [CrossRef] [PubMed]

12. Andia, I.; Maffulli, N. Biological therapies in regenerative sports medicine. Sports Med. 2017, 47, 807-828. [CrossRef] [PubMed]

13. Dash, N.R.; Dash, S.N.; Routray, P.; Mohapatra, S.; Mohapatra, P.C. Targeting nonhealing ulcers of lower extremity in human through autologous bone marrow-derived mesenchymal stem cells. Rejuvenation Res. 2009, 12, 359-366. [CrossRef] [PubMed]

14. Dubsky, M.; Jirkovska, A.; Bem, R.; Fejfarova, V.; Pagacova, L.; Sixta, B.; Varga, M.; Langkramer, S.; Sykova, E.; Jude, E.B. Both autologous bone marrow mononuclear cell and peripheral blood progenitor cell therapies similarly improve ischaemia in patients with diabetic foot in comparison with control treatment. Diabetes Metab. Res. Rev. 2013, 29, 369-376. [CrossRef] [PubMed]

15. Han, S.K.; Kim, H.R.; Kim, W.K. The treatment of diabetic foot ulcers with uncultured, processed lipoaspirate cells: A pilot study. Wound Repair Regen. 2010, 18, 342-348. [CrossRef] [PubMed]

16. Jain, P.; Perakath, B.; Jesudason, M.R.; Nayak, S. The effect of autologous bone marrow-derived cells on healing chronic lower extremity wounds: Results of a randomized controlled study. Ostomy Wound Manag. 2011, 57, 38-44.

17. Kirana, S.; Stratmann, B.; Prante, C.; Prohaska, W.; Koerperich, H.; Lammers, D.; Gastens, M.H.; Quast, T.; Negrean, M.; Stirban, O.A.; et al. Autologous stem cell therapy in the treatment of limb ischaemia induced chronic tissue ulcers of diabetic foot patients. Int. J. Clin. Pract. 2012, 66, 384-393. [CrossRef] [PubMed]

18. Lu, D.; Chen, B.; Liang, Z.; Deng, W.; Jiang, Y.; Li, S.; Xu, J.; Wu, Q.; Zhang, Z.; Xie, B.; et al. Comparison of bone marrow mesenchymal stem cells with bone marrow-derived mononuclear cells for treatment of diabetic critical limb ischemia and foot ulcer: A double-blind, randomized, controlled trial. Diabetes Res. Clin. Pract. 2011, 92, 26-36. [CrossRef] [PubMed]

19. Marino, G.; Moraci, M.; Armenia, E.; Orabona, C.; Sergio, R.; De Sena, G.; Capuozzo, V.; Barbarisi, M.; Rosso, F.; Giordano, G.; et al. Therapy with autologous adipose-derived regenerative cells for the care of chronic ulcer of lower limbs in patients with peripheral arterial disease. J. Surg. Res. 2013, 185, 36-44. [CrossRef] [PubMed]

20. Prochazka, V.; Gumulec, J.; Jaluvka, F.; Salounova, D.; Jonszta, T.; Czerny, D.; Krajca, J.; Urbanec, R.; Klement, P.; Martinek, J.; et al. Cell therapy, a new standard in management of chronic critical limb ischemia and foot ulcer. Cell Transplant. 2010, 19, 1413-1424. [CrossRef] [PubMed]

21. Raposio, E.; Bertozzi, N.; Bonomini, S.; Bernuzzi, G.; Formentini, A.; Grignaffini, E.; Pio Grieco, M. Adipose-derived stem cells added to platelet-rich plasma for chronic skin ulcer therapy. Wounds Compend. Clin. Res. Pract. 2016, 28, 126-131.

22. Walter, D.H.; Krankenberg, H.; Balzer, J.O.; Kalka, C.; Baumgartner, I.; Schluter, M.; Tonn, T.; Seeger, F.; Dimmeler, S.; Lindhoff-Last, E.; et al. Intraarterial administration of bone marrow mononuclear cells in patients with critical limb ischemia: A randomized-start, placebo-controlled pilot trial (PROVASA). Circ. Cardiovasc. Interv. 2011, 4, 26-37. [CrossRef] [PubMed]

23. Ahmed, M.; Reffat, S.A.; Hassan, A.; Eskander, F. Platelet-rich plasma for the treatment of clean diabetic foot ulcers. Ann. Vasc. Surg. 2017, 38, 206-211. [CrossRef] [PubMed]

24. Anitua, E.; Aguirre, J.J.; Algorta, J.; Ayerdi, E.; Cabezas, A.I.; Orive, G.; Andia, I. Effectiveness of autologous preparation rich in growth factors for the treatment of chronic cutaneous ulcers. J. Biomed. Mater. Res. Part $B$ Appl. Biomater. 2008, 84, 415-421. [CrossRef] [PubMed]

25. Danielsen, P.; Jorgensen, B.; Karlsmark, T.; Jorgensen, L.N.; Agren, M.S. Effect of topical autologous platelet-rich fibrin versus no intervention on epithelialization of donor sites and meshed split-thickness skin autografts: A randomized clinical trial. Plast. Reconstr. Surg. 2008, 122, 1431-1440. [CrossRef] [PubMed]

26. Driver, V.R.; Hanft, J.; Fylling, C.P.; Beriou, J.M. A prospective, randomized, controlled trial of autologous platelet-rich plasma gel for the treatment of diabetic foot ulcers. Ostomy Wound Manag. 2006, 52, 68-70. 
27. Jeong, S.H.; Han, S.K.; Kim, W.K. Treatment of diabetic foot ulcers using a blood bank platelet concentrate. Plast. Reconstr. Surg. 2010, 125, 944-952. [CrossRef] [PubMed]

28. Kakagia, D.D.; Kazakos, K.J.; Xarchas, K.C.; Karanikas, M.; Georgiadis, G.S.; Tripsiannis, G.; Manolas, C. Synergistic action of protease-modulating matrix and autologous growth factors in healing of diabetic foot ulcers. A prospective randomized trial. J. Diabetes Complicat. 2007, 21, 387-391. [CrossRef] [PubMed]

29. Karimi, R.; Afshar, M.; Salimian, M.; Sharif, A.; Hidariyan, M. The effect of platelet rich plasma dressing on healing diabetic foot ulcers. Nurs. Midwifery Stud. 2016, 5. [CrossRef]

30. Knighton, D.R.; Ciresi, K.; Fiegel, V.D.; Schumerth, S.; Butler, E.; Cerra, F. Stimulation of repair in chronic, nonhealing, cutaneous ulcers using platelet-derived wound healing formula. Surg. Gynecol. Obstet. 1990, 170, 56-60. [PubMed]

31. Krupski, W.C.; Reilly, L.M.; Perez, S.; Moss, K.M.; Crombleholme, P.A.; Rapp, J.H. A prospective randomized trial of autologous platelet-derived wound healing factors for treatment of chronic nonhealing wounds: A preliminary report. J. Vasc. Surg. 1991, 14, 526-532. [CrossRef]

32. Li, L.; Chen, D.; Wang, C.; Yuan, N.; Wang, Y.; He, L.; Yang, Y.; Chen, L.; Liu, G.; Li, X.; et al. Autologous platelet-rich gel for treatment of diabetic chronic refractory cutaneous ulcers: A prospective, randomized clinical trial. Wound Repair Regen. 2015, 23, 495-505. [CrossRef] [PubMed]

33. Moneib, H.A.; Youssef, S.S.; Aly, D.G.; Rizk, M.A.; Abdelhakeem, Y.I. Autologous platelet-rich plasma versus conventional therapy for the treatment of chronic venous leg ulcers: A comparative study. J. Cosmet. Dermatol. 2017. [CrossRef] [PubMed]

34. Obolenskiy, V.N.; Ermolova, D.A.; Laberko, L.A. Clinical and economic effectiveness of the use of platelet-rich plasma in the treatment of chronic wounds. Wound Med. 2017, 19, 27-32. [CrossRef]

35. Pravin, A.J.S.; Sridhar, V.; Srinivasan, B.N. Autologous platelet rich plasma (PRP) versus leucocyte-platelet rich fibrin (L-PRF) in chronic non-healing leg ulcers-A randomised, open labelled, comparative study. J. Evol. Med. Dent. Sci. 2016, 5, 7460. [CrossRef] [PubMed]

36. Saad Setta, H.; Elshahat, A.; Elsherbiny, K.; Massoud, K.; Safe, I. Platelet-rich plasma versus platelet-poor plasma in the management of chronic diabetic foot ulcers: A comparative study. Int. Wound J. 2011, 8, 307-312. [CrossRef] [PubMed]

37. Saldalamacchia, G.; Lapice, E.; Cuomo, V.; De Feo, E.; D’Agostino, E.; Rivellese, A.A.; Vaccaro, O. A controlled study of the use of autologous platelet gel for the treatment of diabetic foot ulcers. Nutr. Metab. Cardiovasc. Dis. 2004, 14, 395-396. [CrossRef]

38. Senet, P.; Bon, F.X.; Benbunan, M.; Bussel, A.; Traineau, R.; Calvo, F.; Dubertret, L.; Dosquet, C. Randomized trial and local biological effect of autologous platelets used as adjuvant therapy for chronic venous leg ulcers. J. Vasc. Surg. 2003, 38, 1342-1348. [CrossRef]

39. Stacey, M.C.; Mata, S.D.; Trengove, N.J.; Mather, C.A. Randomised double-blind placebo controlled trial of topical autologous platelet lysate in venous ulcer healing. Eur. J. Vasc. Endovasc. Surg. Off. J. Eur. Soc. Vasc. Surg. 2000, 20, 296-301. [CrossRef] [PubMed]

40. Steed, D.L.; Goslen, J.B.; Holloway, G.A.; Malone, J.M.; Bunt, T.J.; Webster, M.W. Randomized prospective double-blind trial in healing chronic diabetic foot ulcers. Ct-102 activated platelet supernatant, topical versus placebo. Diabetes Care 1992, 15, 1598-1604. [CrossRef] [PubMed]

41. Shin, J.Y.; Roh, S.G.; Sharaf, B.; Lee, N.H. Risk of major limb amputation in diabetic foot ulcer and accompanying disease: A meta-analysis. J. Plast. Reconstruct. Aesthet. Surg. 2017, 70, 1681-1688. [CrossRef] [PubMed]

42. Fadini, G.P.; Agostini, C.; Avogaro, A. Autologous stem cell therapy for peripheral arterial disease meta-analysis and systematic review of the literature. Atherosclerosis 2010, 209, 10-17. [CrossRef] [PubMed]

43. Teraa, M.; Sprengers, R.W.; van der Graaf, Y.; Peters, C.E.; Moll, F.L.; Verhaar, M.C. Autologous bone marrow-derived cell therapy in patients with critical limb ischemia: A meta-analysis of randomized controlled clinical trials. Ann. Surg. 2013, 258, 922-929. [CrossRef] [PubMed]

44. Catherine Foss, S.R.; Ambrose, J.; Carney, J.; Nilsson, M. International Federation for Adipose Therapeutics and Science. Available online: http:/ / www.ifats.org/ (accessed on 4 October 2017).

45. Dominici, M.; Le Blanc, K.; Mueller, I.; Slaper-Cortenbach, I.; Marini, F.; Krause, D.; Deans, R.; Keating, A.; Prockop, D.; Horwitz, E. Minimal criteria for defining multipotent mesenchymal stromal cells. The international society for cellular therapy position statement. Cytotherapy 2006, 8, 315-317. [CrossRef] [PubMed] 
46. Zuk, P.A.; Zhu, M.; Mizuno, H.; Huang, J.; Futrell, J.W.; Katz, A.J.; Benhaim, P.; Lorenz, H.P.; Hedrick, M.H. Multilineage cells from human adipose tissue: Implications for cell-based therapies. Tissue Eng. 2001, 7 , 211-228. [CrossRef] [PubMed]

47. Dimarino, A.M.; Caplan, A.I.; Bonfield, T.L. Mesenchymal stem cells in tissue repair. Front. Immunol. 2013, 4, 201. [CrossRef] [PubMed]

48. Centeno, C.J.; Al-Sayegh, H.; Freeman, M.D.; Smith, J.; Murrell, W.D.; Bubnov, R. A multi-center analysis of adverse events among two thousand, three hundred and seventy two adult patients undergoing adult autologous stem cell therapy for orthopaedic conditions. Int. Orthop. 2016, 40, 1755-1765. [CrossRef] [PubMed]

49. Pak, J.; Chang, J.J.; Lee, J.H.; Lee, S.H. Safety reporting on implantation of autologous adipose tissue-derived stem cells with platelet-rich plasma into human articular joints. BMC Musculoskelet. Disord. 2013, $14,337$. [CrossRef] [PubMed]

50. Peeters, C.M.; Leijs, M.J.; Reijman, M.; van Osch, G.J.; Bos, P.K. Safety of intra-articular cell-therapy with culture-expanded stem cells in humans: A systematic literature review. Osteoarthr. Cartil. 2013, 21, 1465-1473. [CrossRef] [PubMed]

51. Knighton, D.R.; Ciresi, K.F.; Fiegel, V.D.; Austin, L.L.; Butler, E.L. Classification and treatment of chronic nonhealing wounds. Successful treatment with autologous platelet-derived wound healing factors (PDWHF). Ann. Surg. 1986, 204, 322-330. [CrossRef] [PubMed]

52. Andia, I.; Abate, M. Platelet-rich plasma: Combinational treatment modalities for musculoskeletal conditions. Front. Med. 2017. [CrossRef] [PubMed]

53. Andia, I.; Maffulli, N. Muscle and tendon injuries: The role of biological interventions to promote and assist healing and recovery. Arthrosc. J. Arthrosc. Relat. Surg. Off. Publ. Arthrosc. Assoc. N. Am. Int. Arthrosc. Assoc. 2015, 31, 999-1015. [CrossRef] [PubMed]

54. Anitua, E.; Sanchez, M.; Zalduendo, M.M.; de la Fuente, M.; Prado, R.; Orive, G.; Andia, I. Fibroblastic response to treatment with different preparations rich in growth factors. Cell Prolif. 2009, 42, 162-170. [CrossRef] [PubMed]

55. Nurden, A.T.; Nurden, P.; Sanchez, M.; Andia, I.; Anitua, E. Platelets and wound healing. Front. Biosci. 2008, 13, 3532-3548. [CrossRef] [PubMed]

56. Carter, M.J.; Fylling, C.P.; Parnell, L.K. Use of platelet rich plasma gel on wound healing: A systematic review and meta-analysis. Eplasty 2011, 11, e38. [PubMed]

57. Martinez-Zapata, M.J.; Marti-Carvajal, A.J.; Sola, I.; Exposito, J.A.; Bolibar, I.; Rodriguez, L.; Garcia, J.; Zaror, C. Autologous platelet-rich plasma for treating chronic wounds. Cochrane Database Syst. Rev. 2016. [CrossRef]

58. Murray, I.R.; Geeslin, A.G.; Goudie, E.B.; Petrigliano, F.A.; LaPrade, R.F. Minimum information for studies evaluating biologics in orthopaedics (MIBO): Platelet-rich plasma and mesenchymal stem cells. J. Bone Jt. Surg. Am. Vol. 2017, 99, 809-819. [CrossRef] [PubMed]

59. Carter, M.J.; Fylling, C.P.; Li, W.W.; de Leon, J.; Driver, V.R.; Serena, T.E.; Wilson, J. Analysis of run-in and treatment data in a wound outcomes registry: Clinical impact of topical platelet-rich plasma gel on healing trajectory. Int. Wound J. 2011, 8, 638-650. [CrossRef] [PubMed]

60. De Leon, J.M.; Driver, V.R.; Fylling, C.P.; Carter, M.J.; Anderson, C.; Wilson, J.; Dougherty, R.M.; Fuston, D.; Trigilia, D.; Valenski, V.; et al. The clinical relevance of treating chronic wounds with an enhanced near-physiological concentration of platelet-rich plasma gel. Adv. Skin Wound Care 2011, 24, 357-368. [CrossRef] [PubMed]

61. Margolis, D.J.; Kantor, J.; Santanna, J.; Strom, B.L.; Berlin, J.A. Effectiveness of platelet releasate for the treatment of diabetic neuropathic foot ulcers. Diabetes Care 2001, 24, 483-488. [CrossRef] [PubMed]

62. Futrega, K.; King, M.; Lott, W.B.; Doran, M.R. Treating the whole not the hole: Necessary coupling of technologies for diabetic foot ulcer treatment. Trends Mol. Med. 2014, 20, 137-142. [CrossRef] [PubMed]

63. Wang, J.H.; Zhao, K.; Liu, H.L.; Zhao, H.M.; Yang, J.; Sun, X.K. Infected bone inactivation combined with transplantation of autologous platelet-rich plasma and bone marrow for treatment of chronic osteomyelitis. Eur. Rev. Med. Pharmacol. Sci. 2015, 19, 4488-4493. [PubMed]

64. Morimoto, N.; Kakudo, N.; Matsui, M.; Ogura, T.; Hara, T.; Suzuki, K.; Yamamoto, M.; Tabata, Y.; Kusumoto, K. Exploratory clinical trial of combination wound therapy with a gelatin sheet and platelet-rich plasma in patients with chronic skin ulcers: Study protocol. BMJ Open 2015, 5. [CrossRef] [PubMed] 
65. Perez-Zabala, E.; Basterretxea, A.; Larrazabal, A.; Perez-Del-Pecho, K.; Rubio-Azpeitia, E.; Andia, I. Biological approach for the management of non-healing diabetic foot ulcers. J. Tissue Viability 2016, 25, 157-163. [CrossRef] [PubMed]

66. Higgins, J.P.T. Cochrane Handbook for Systematic Reviews of Interventions, Version 5.1.0. Available online: www.cochrane-handbook.org (accessed on 9 October 2017).

67. PRISMA. Preferred reporting items for systematic reviews and meta-analysis. Syst. Rev. 2015, 4, 1.

68. MEDLINE. Available online: https:/ / www.ncbi.nlm.nih.gov/pubmed (accessed on 30 July 2017).

69. EMBASE. Available online: http:/ / ovidsp.uk.ovid.com/sp-3.27.1a/ovidweb.cgi (accessed on 30 July 2017).

70. The Cochrane Library Clinical Trials Database. Available online: http://www.cochranelibrary.com/ (accessed on 30 July 2017).

2017 by the authors. Licensee MDPI, Basel, Switzerland. This article is an open access article distributed under the terms and conditions of the Creative Commons Attribution (CC BY) license (http://creativecommons.org/licenses/by/4.0/). 